\title{
Effect of mechanical treatment on the powder properties of zinc phosphate white pigments
}

\author{
Hiroaki ONODA*, Miho HARUKI \\ Department of Informatics and Environmental Sciences, Kyoto Prefectural University, 1-5, \\ Shimogamo Nakaragi-cyo, Sakyo-ku, Kyoto 606-8522, Japan
}

Received: May 13, 2015; Revised: August 03, 2015; Accepted: August 12, 2015

(C) The Author(s) 2015. This article is published with open access at Springerlink.com

\begin{abstract}
Zinc oxide, which has photocatalytic activity, is used as a white pigment for cosmetics, resulting in a certain amount of sebum on the skin to be decomposed by the ultraviolet radiation in the sunlight. In this work, zinc phosphates as novel white pigments for use in cosmetics were prepared from zinc nitrate and sodium dihydrogen phosphate, and then ball-milled under various conditions. The chemical composition, powder properties, photocatalytic activity, color phase, moisture retention, and smoothness of the zinc phosphates were studied. The zinc phosphate particle size was decreased by mechanical treatment. In particular, the sample treated with sodium lactate solution had much smaller particles. The milled zinc phosphates exhibited less photocatalytic activity than zinc oxide, and thus should not decompose sebum on the skin. The milled zinc phosphates showed sufficiently high reflectance within the range of visible light to act as novel white pigments. The sample treated with sodium lactate solution had higher water retention than the sample treated with water. Further, the slip resistance and roughness of the powder particles decreased as a result of treatment with sodium lactate solution.
\end{abstract}

Keywords: white pigment; zinc phosphate; mechanical treatment; photocatalytic activity; water retention

\section{Introduction}

As white pigments, titanium dioxide and zinc oxide are used for cosmetic applications [1]. These oxides are well known to exhibit photocatalytic activity. Therefore, when these compounds are included in cosmetics, a certain amount of sebum on the skin is decomposed by the ultraviolet radiation in the sunlight. To repress this effect, several technical processes have been investigated and used, including composite particles with silicon oxide [2]. However, such

\footnotetext{
* Corresponding author.

E-mail: onoda@kpu.ac.jp
}

materials are too hard for use on the human face. Instead, soft materials are required for use as white facial pigments. In addition, one report found that micro fine oxides are absorbed through the skin [3]. Therefore, novel white pigments that are not absorbed by the skin and do not exhibit photocatalytic activity should be developed to protect the skin.

Phosphates have been used as ceramic materials, catalysts, fluorescent materials, dielectric substances, metal-surface treatments, detergents, food additives, fuel cells, pigments, etc. $[4,5]$. Phosphate materials are well known to have a high affinity for living organisms and are therefore expected to be useful as white pigments in cosmetics. 
When used as cosmetic pigments, the particle shape and size distribution of the phosphate particles are important. Homogeneous spherical particles are expected to spread well on the skin. However, overly small particles are unsuitable because the pigments might enter the pores of the skin. Generally, sub-micrometer size pigments are used. In the earlier studies [6-8], we prepared titanium and zinc phosphate pigments with no catalytic activity. However, zinc phosphates had particle sizes larger than $1 \mu \mathrm{m}$ [8]; therefore, a process to obtain small zinc phosphate particles is required. For inorganic materials, it is well known that mechanical treatment has an influence on particle properties $[9,10]$. Therefore, the present work is undertaken to obtain homogeneous zinc phosphate particles by mechanical treatment.

The moisture retention of materials used in cosmetics is important for preventing dry skin. Sodium lactate and glycerin improve the moisture retention of materials and are therefore generally used in cosmetic products. The addition of sodium lactate is effective for obtaining spherical particles and moisture retention with zinc phosphates [8]. Therefore, in this work, sodium lactate and glycerin were used as milling media.

In this work, as a novel white pigment, zinc phosphate was prepared from zinc nitrate and sodium dihydrogen phosphate, and then ball-milled under various conditions. To obtain homogeneous small particles, the milling time and media were studied. The chemical composition, powder properties, photocatalytic activity, color phase, moisture retention, and smoothness of the milled materials were studied for applications in cosmetics.

\section{Experimental}

Solutions of zinc nitrate $(0.1 \mathrm{~mol} / \mathrm{L})$ and sodium dihydrogen phosphate $(0.1 \mathrm{~mol} / \mathrm{L})$ were mixed in a $\mathrm{Zn} / \mathrm{P}$ molar ratio of 3:2 at room temperature for more than $1 \mathrm{~h}$. The mixed solution was adjusted with ammonia solution to $\mathrm{pH}=5$. The precipitates were then filtered off, washed with water, and dried at room temperature over 3 days. All chemicals used in this work were of commercial purity from Wako Chemical Industries Ltd. (Osaka, Japan) and used without further purification.

The samples $(2 \mathrm{~g})$ were placed in a polypropylene pot $(38 \mathrm{~mL})$ with 6 mullite balls (average weight $1.66 \mathrm{~g}$, diameter $10 \mathrm{~mm}$ ). This pot was rotated with a ball-mill (Nitto Ball Mill ANZ-10S, Nitto Kagaku Co., Ltd.) at $190 \mathrm{rpm}$ for $12,24,36$, and $48 \mathrm{~h}$. To clarify the effect of the milling media, the samples $(2 \mathrm{~g})$ were treated with $10 \mathrm{~mL}$ of water for $12,24,36$, and $48 \mathrm{~h}$, or with $10 \mathrm{~mL}$ of ethanol for 12 and $24 \mathrm{~h}$. Mixed solutions $(10 \mathrm{~mL})$ prepared from $70 \mathrm{wt} \%$ sodium lactate and water or from glycerin and water $(1: 9,3: 7$, and 5:5) were used as the milling media for $12 \mathrm{~h}$. The milled samples with solvents were filtered off and dried over $24 \mathrm{~h}$.

The crystalline phases of these materials were analyzed using X-ray diffraction (XRD). The XRD patterns were recorded on an X-ray diffractometer (MiniFlex, Rigaku Corp.) using monochromated $\mathrm{Cu}$ $\mathrm{K} \alpha$ radiation. The particle shape and size of the zinc phosphate precipitates were estimated based on scanning electron microscopy (SEM; JGM-5510LV, JEOL Ltd.) images. The particle size distributions of these materials were measured using a centrifugal precipitation particle-size analyzer (SA-CP3L, Shimadzu Corp.).

The cosmetic properties of these materials were estimated according to the photocatalytic activity, color phase, moisture retention, and smoothness. The photocatalytic activity of the samples was estimated using the decomposition of methylene blue under irradiation at $365 \mathrm{~nm}$ [11-13]. The decomposition of methylene blue was estimated using the absorption at $660 \mathrm{~nm}$ (UV2100, Shimadzu Corp.). The color phase of the phosphate pigments was estimated from ultraviolet-visible (UV-Vis) reflectance spectra (UV2100, Shimadzu Corp.; reference compound $\mathrm{BaSO}_{4}$ ). To determine the moisture retention of the samples, $0.3 \mathrm{~g}$ of the samples was mixed with $0.1 \mathrm{~g}$ of water, and the weight loss was evaluated at $50{ }^{\circ} \mathrm{C}$ (MS-70 Moisture Analyzer, A\&D Instruments Co. Ltd.; relative humidity $50 \%-60 \%$ ). The same weight loss over a longer time indicates higher water retention in a sample. The particle smoothness was measured on artificial leather based on a KES-SE objective evaluation of the surface friction properties (Kato Tech Co., Ltd.). The MIU (average value of $\mu$ over a distance of $20 \mathrm{~mm}$ ) and MMD (degree of fluctuation in MIU) values represent the slip resistance (coefficient of kinetic friction) and roughness of the powders (dispersion of the coefficient of kinetic friction), respectively. The sample powders were spread onto the leather (load of sample, about $50 \mathrm{mg}$ ). A sensor was run over the powders (scan speed $1 \mathrm{~mm} / \mathrm{s}$, area scanned $3 \mathrm{~cm}^{2}$ ), and the MIU and MMD values were calculated from the power required to move the sensor 
and the pitch of the sensor, respectively. The MIU and MMD have no units because they are related to the coefficient of friction and scattering, respectively. The dispersions of the MIU and MMD values were 0.03 and 0.002 , respectively, for three measurements.

\section{Results and discussion}

\section{1 Crystalline phase and powder properties of zinc phosphates}

Figure 1 shows the XRD patterns of the samples treated for various time without milling media. All the samples have peaks corresponding to zinc phosphate tetrahydrate, $\mathrm{Zn}_{3}\left(\mathrm{PO}_{4}\right)_{2} \cdot 4 \mathrm{H}_{2} \mathrm{O}$ [14]. The strongest peak in the sample without milling appears at $\sim 19.5^{\circ}$; on the other hand, the treated samples have the strongest peaks at $\sim 31.5^{\circ}$. By milling, the crystalline phase corresponding to $19.5^{\circ}$ decreases.

Figure 2 shows SEM images of the samples treated under various conditions. The sample without milling have plate particles (Fig. 2(a)). Through treatment without milling media, the plate particles become smaller (Fig. 2(b)). The samples treated with water, sodium lactate aqueous solution, and glycerin aqueous solution have smaller particles than the samples treated without milling media.

Small and homogeneous particles are suitable for cosmetic applications. However, overly small particles have a major shortcoming in that they may enter the pores of the skin [3]. Generally, sub-micrometer sized pigments are used. A standard size of white pigment

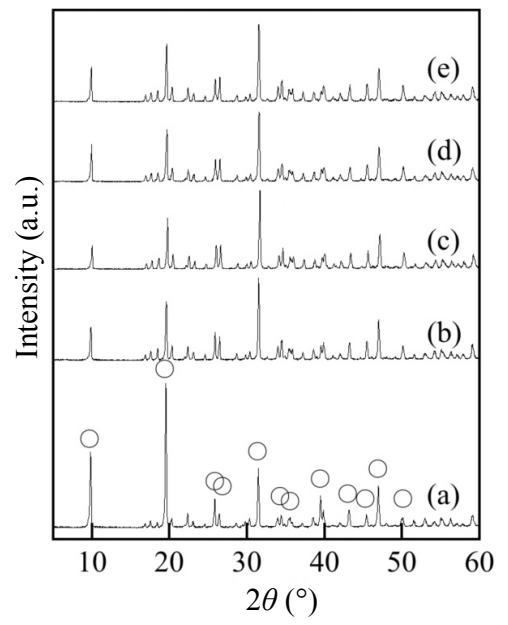

Fig. 1 XRD patterns of the samples treated for various time (without milling media): (a) 0, (b) 12, (c) 24, (d) 36, and (e) 48 h. $\circ: \mathrm{Zn}_{3}\left(\mathrm{PO}_{4}\right)_{2} \cdot 4 \mathrm{H}_{2} \mathrm{O}$. particles for use in cosmetics is difficult to determine because skin pore sizes are affected by factors such as age, gender, and climate. Furthermore, overly large particles are inappropriate because their coatings tend to crack on the skin. It is therefore important to control the pigment particle size. Figure 3 shows the particle size distributions of the samples treated without milling media for various time. The sample without mechanical treatment has particles larger than $10 \mu \mathrm{m}$. Mechanical treatment increases the amount of particles with sizes smaller than $10 \mu \mathrm{m}$. These results correspond with the results from the SEM images. Figure 4 shows the particle size distributions of the samples treated with various milling media. The sample treated with ethanol has a slightly larger particle size distribution than the other samples. On the other hand, the samples treated with water, sodium lactate solution, and glycerin solution have smaller particles than the sample treated without milling media. In particular, the sample treated with sodium lactate solution has much smaller particles than the other samples. Sodium lactate solution is effective as a milling media to prevent the aggregation of particles.

\section{2 Cosmetic properties of milled phosphates}

Figure 5 shows the photocatalytic activities of the samples treated under various conditions. Because it is used as a white pigment in cosmetics, zinc oxide was evaluated for comparison with zinc phosphate [1]. Methylene blue is decomposed in the presence of zinc oxide under UV irradiation (Fig. 5(h)). On the other hand, zinc phosphate shows little photocatalytic activity irrespective of the milling time or media (Figs. 5(b)-5(g)), which indicates zinc phosphate is a mild material that can protect sebum on the skin. This is an importance characteristic of zinc phosphate as a novel white pigment.

Figure 6 shows the UV-Vis reflectance spectra of the zinc phosphates treated under various conditions. All samples show high reflectance within the range of visible light irrespective of the milling time or media. All samples obtained in this work are sufficient as white powders.

Moisture helps to prevent itchiness and damage to the skin. It is important that the pigments used in cosmetics retain moisture on the skin. Figure 7 shows the moisture retention of the samples treated with various concentrations of sodium lactate. At the same retention time, a smaller weight loss indicates higher moisture retention of the phosphate pigments. For 

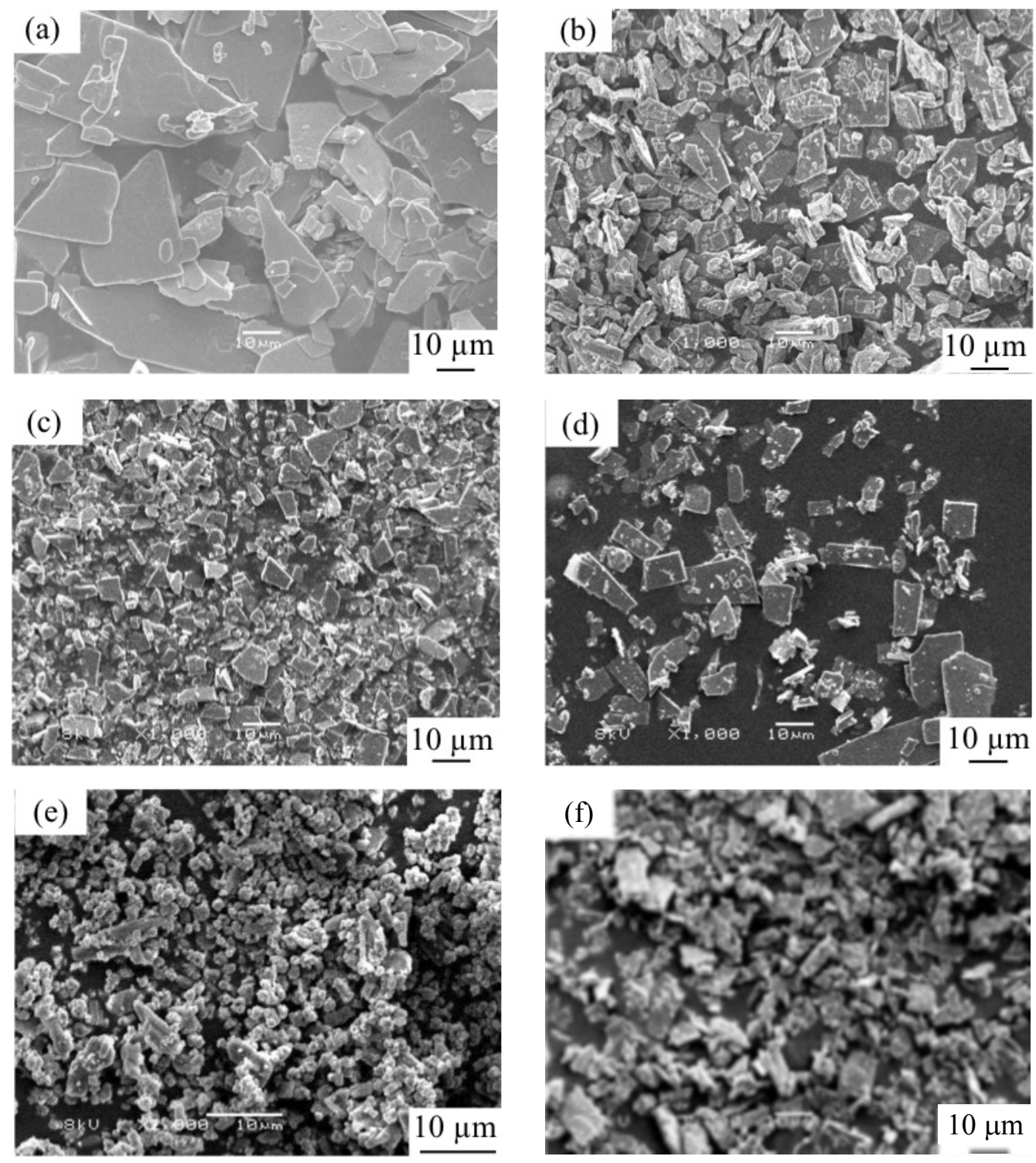

Fig. 2 SEM images of the samples treated under various conditions: (a) untreated, (b) without milling media for $12 \mathrm{~h}$, (c) with water for $12 \mathrm{~h},(\mathrm{~d})$ with ethanol for $12 \mathrm{~h}$, (e) with 5:5 sodium lactate aqueous solution for $12 \mathrm{~h}$, and (e) with 5:5 glycerin aqueous solution for $12 \mathrm{~h}$.

example, at $10 \mathrm{~min}$, the sample treated with $0 \%$ sodium lactate solution (namely water) has a $14.7 \%$ weight loss (Fig. 7(a)), whereas the sample treated with 3:7 sodium lactate solution $(3 \mathrm{~mL}$ sodium lactate $/ 7 \mathrm{~mL}$ water) has an $11.7 \%$ weight loss (Fig. 7(c)). The sample treated with 3:7 sodium lactate solution has higher water retention than the sample treated with water. Sodium lactate is effective for improving the moisture retention. In the previous work [15], the addition of sodium lactate also improves the moisture retention of zinc phosphate. This improvement is caused by the inherent water retention characteristic of sodium lactate and the easy incorporation of sodium lactate into zinc phosphate materials. These zinc phosphate white pigments with moisture retention are suitable for cosmetic applications.

As described above, a pigment with a high level of smoothness spreads well across the skin, and powder smoothness is important for use in cosmetics [16]. Table 1 shows the smoothness of the samples prepared under various conditions. Generally, for cosmetic applications, suitable MIU and MMD values are lower than 0.6 and 0.04 , respectively. The sample treated with ethanol has a higher MIU value than the other samples, whereas the sample treated with 5:5 sodium 


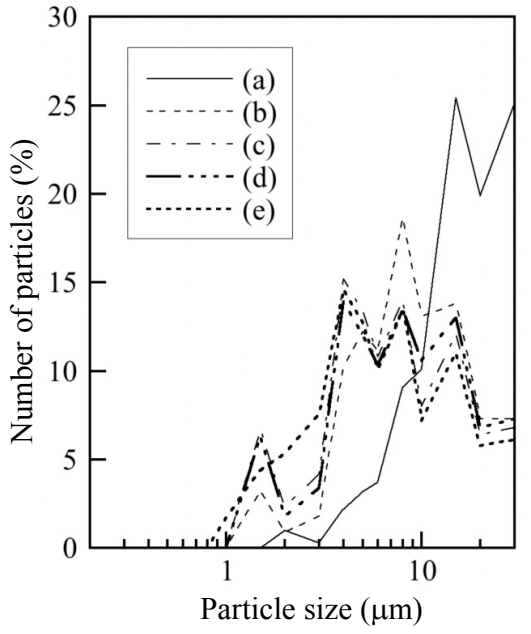

Fig. 3 Particle size distributions of the samples treated for various time (without milling media): (a) 0 , (b) 12 , (c) 24 , (d) 36 , and (e) $48 \mathrm{~h}$.

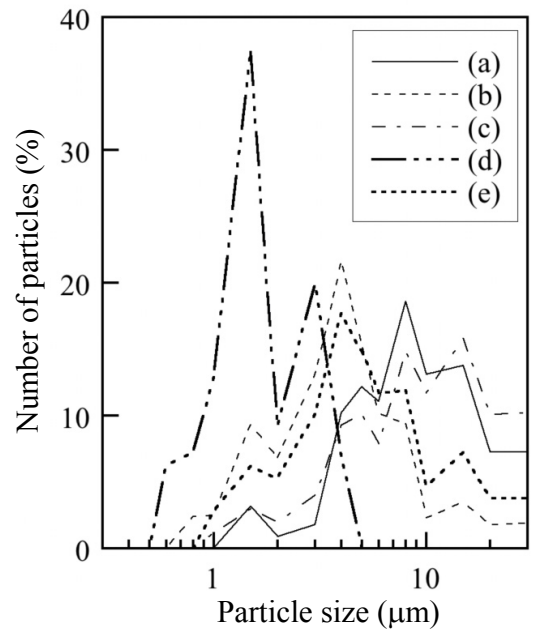

Fig. 4 Particle size distributions of the samples treated with various milling media for $12 \mathrm{~h}$ : (a) no milling media, (b) water, (c) ethanol, (d) 5:5 sodium lactate aqueous solution, and (e) 5:5 glycerin aqueous solution.

Table 1 Smoothness of the samples treated under various conditions

\begin{tabular}{|c|c|c|c|c|c|c|}
\hline \multirow{2}{*}{$\begin{array}{l}\text { Time } \\
\text { (h) }\end{array}$} & \multicolumn{4}{|c|}{ Solvent $(\mathrm{mL})$} & \multirow{2}{*}{ MIU } & \multirow{2}{*}{ MMD } \\
\hline & Water & Ethanol & Glycerin & Sodium lactate & & \\
\hline 0 & 0 & 0 & 0 & 0 & 1.037 & 0.011 \\
\hline 12 & 0 & 0 & 0 & 0 & 1.107 & 0.011 \\
\hline 36 & 0 & 0 & 0 & 0 & 1.013 & 0.011 \\
\hline 12 & 10 & 0 & 0 & 0 & 1.043 & 0.011 \\
\hline 12 & 0 & 10 & 0 & 0 & 1.140 & 0.012 \\
\hline 12 & 5 & 0 & 5 & 0 & 1.057 & 0.007 \\
\hline 12 & 5 & 0 & 0 & 5 & 0.727 & 0.011 \\
\hline
\end{tabular}

lactate solution ( $5 \mathrm{~mL}$ sodium lactate $/ 5 \mathrm{~mL}$ water) has a lower MIU value. As well as decreasing the particle size and improving moisture retention, sodium lactate is effective for improving the smoothness of zinc phosphate.

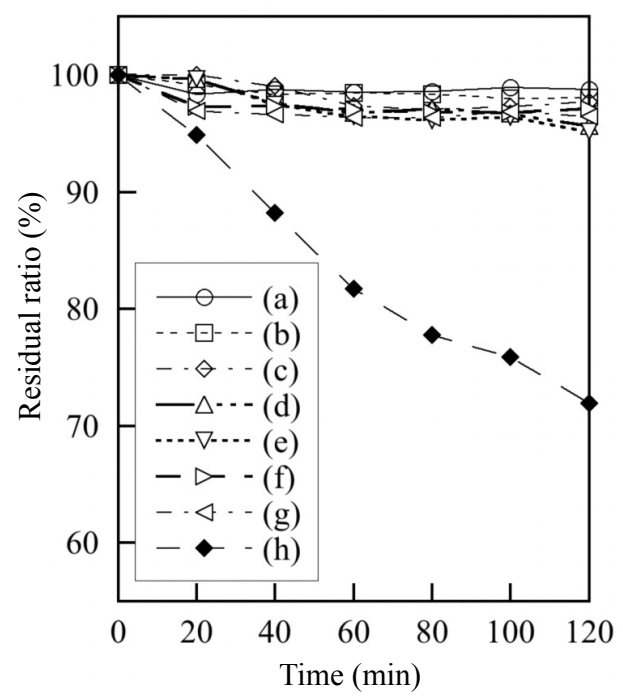

Fig. 5 Photocatalytic activities of the samples treated under various conditions: (a) blank, (b) untreated zinc phosphate powders, (c) without milling media for $48 \mathrm{~h}$, (d) with water for $48 \mathrm{~h}$, (e) with ethanol for $24 \mathrm{~h}$, (f) with 5:5 sodium lactate aqueous solution for $12 \mathrm{~h}$, (g) with 5:5 glycerin aqueous solution for $12 \mathrm{~h}$. (h) $\mathrm{ZnO}$.

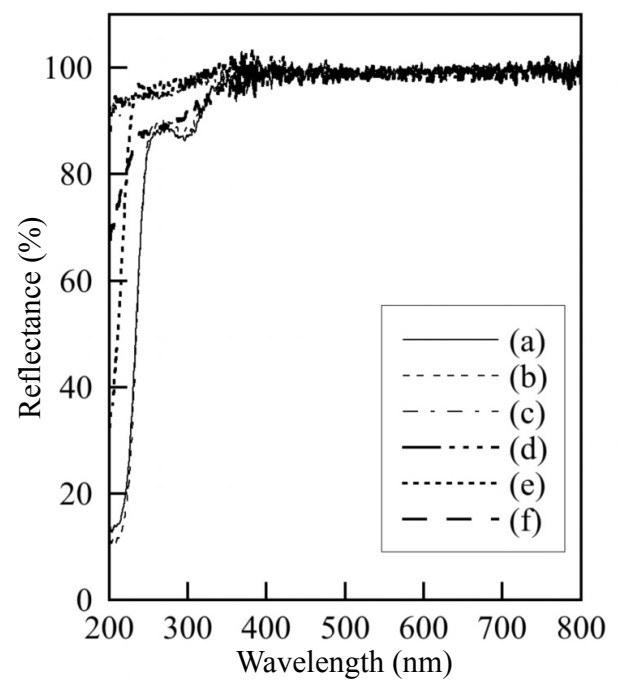

Fig. 6 UV-Vis reflectance of the samples treated under various conditions: (a) untreated zinc phosphate powders, (b) without milling media for $48 \mathrm{~h}$, (c) with water for $48 \mathrm{~h}$, (d) with ethanol for $24 \mathrm{~h}$, (e) with 5:5 sodium lactate aqueous solution for $12 \mathrm{~h}$, and (f) with 5:5 glycerin aqueous solution for $12 \mathrm{~h}$.

\section{Conclusions}

Zinc phosphates were obtained from zinc nitrate and sodium dihydrogen phosphate solutions, and then ball-milled. Water, ethanol, sodium lactate solution, and glycerin solution were used as milling media. The 


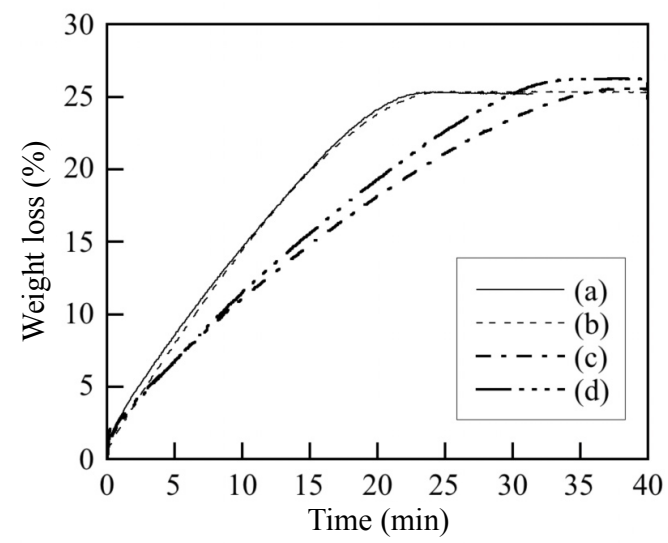

Fig. 7 Water retention of the samples treated with various concentrations of sodium lactate for $12 \mathrm{~h}$ : (a) 0:10, (b) 1:9, (c) 3:7, and (d) 5:5 sodium lactate/water.

XRD peak patterns indicated that the samples consisted of zinc phosphate tetrahydrate, and the XRD peaks became weaker following mechanical treatment. The zinc phosphate particle size decreased with mechanical treatment, and the sample treated with sodium lactate solution had much smaller particles than the other samples. The milled zinc phosphates exhibited less photocatalytic activity than zinc oxide, thereby protecting sebum on the skin. The milled zinc phosphates had high reflectance within the range of visible light. The samples treated with sodium lactate solution showed higher water retention than the sample treated with water. Finally, the slip resistance and roughness of the powder particles decreased as a result of treatment with sodium lactate solution.

\section{Acknowledgements}

The support of this work by The Cosmetology Research Foundation is gratefully acknowledged. The authors are grateful to Dr. Takeshi Toyama, Nihon University, Japan, for the smoothness measurements.

Open Access: This article is distributed under the terms of the Creative Commons Attribution License which permits any use, distribution, and reproduction in any medium, provided the original author(s) and the source are credited.

\section{References}

[1] Diebold U. The surface science of titanium dioxide. Surf Sci Rep 2003, 48: 53-229.

[2] Senzui M, Tamura T, Miura K, et al. Study on penetration of titanium dioxide $\left(\mathrm{TiO}_{2}\right)$ nanoparticles into intact and damaged skin in vitro. J Toxicol Sci 2010, 35: 107-113.

[3] Gamer AO, Leibold E, van Ravenzwaay B. The in vitro absorption of microfine zinc oxide and titanium dioxide through porcine skin. Toxicol in Vitro 2006, 20: 301-307.

[4] Jones DJ, Aptel G, Brandhorst M, et al. High surface area mesoporous titanium phosphate: Synthesis and surface acidity determination. J Mater Chem 2000, 10: 1957-1963.

[5] Bhaumik A, Inagaki S. Mesoporous titanium phosphate molecular sieves with ion-exchange capacity. $J$ Am Chem Soc 2001, 123: 691-696.

[6] Onoda H, Yamaguchi T. Influence of ultrasonic treatment on preparation and powder properties of titanium phosphates. J Mater Chem 2012, 22: 19826-19830.

[7] Onoda H, Yamaguchi T, Takenaka A. Synthesis and pigmental properties of titanium phosphate with the addition of urea. Int J Cosmetic Sci 2012, 34: 86-90.

[8] Onoda H, Haruki M, Toyama T. Preparation and powder properties of zinc phosphates with additives. Ceram Int 2014, 40: 3433-3438.

[9] Onoda H, Okumoto K, Tanaka I. Mechanochemical reforming on powder and acidic properties of copper cyclo-tetraphosphates. Mater Chem Phys 2008, 107: 339-343.

[10] Taufiq-Yap YH, Goh CK, Hutchings GJ, et al. Influence of milling media on the physicochemicals and catalytic properties of mechanochemical treated vanadium phosphate catalysts. Catal Lett 2011, 141: 400-407.

[11] Ramaswamy V, Jagtap NB, Vijayanand $\mathrm{S}$, et al. Photocatalytic decomposition of methylene blue on nanocrystalline titania prepared by different methods. Mater Res Bull 2008, 43: 1145-1152.

[12] Du P, Bueno-López A, Verbaas M, et al. The effect of surface OH-population on the photocatalytic activity of rare earth-doped $\mathrm{P} 25-\mathrm{TiO}_{2}$ in methylene blue degradation. $J$ Catal 2008, 206: 75-80.

[13] Mills A. An overview of the methylene blue ISO test for assessing the activities of photocatalytic films. Appl Catal B: Environ 2012, 128: 144-149.

[14] Whitaker A. The crystal structure of hopeite, $\mathrm{Zn}_{3}\left(\mathrm{PO}_{4}\right)_{2} \cdot 4 \mathrm{H}_{2} \mathrm{O}$. Acta Cryst 1975, 31: 2026-2035.

[15] Onoda H, Haruki M, Toyama T. Influence of $\mathrm{pH}$, concentration of sodium lactate as an additive and ultrasonic treatment on synthesis of zinc phosphate white pigments. Int J Cosmetic Sci 2014, 36: 321-326.

[16] Cheng SY, Yuen CWM, Kan CW, et al. A comprehensive study of silicone-based cosmetic textile agent. Fiber Polym 2009, 10: 132-140. 